\title{
The effect of Bi promoter on vanadium phosphate catalysts synthesized via sesquihydrate route.
}

\begin{abstract}
A series of $1 \%, 3 \%$ and $5 \%$ Bi-doped vanadyl pyrophosphate catalysts were prepared via sesquihydrate route (VPOs method). These catalysts were denoted as VPOs-Bi1\%, VPOs-Bi3\% and VPOs-Bi5\%. Bulk and Bi-promoted vanadyl pyrophosphate catalysts prepared via sesquihydrate route exhibited a well-crystallized (VO)2P2O7 phase. Two V5+ phases, i.e. $\beta$ VOPO4 and $\alpha$ II-VOPO4 were observed in all Bi-promoted VPO catalysts, which led to an increase in the specific surface area and average oxidation state of vanadium. Bi-promoted VPO catalysts showed six to nine times higher amounts of oxygen evolved than the bulk VPO catalyst in oxygen TPD and a significant shift in the reduction peaks to lower temperatures. Catalytic tests revealed that both activity and selectivity to maleic anhydride increased with the presence of bismuth promoter.
\end{abstract}

Keyword: Vanadyl pyrophosphate; Bismuth; Selective oxidation. 